\title{
Mild-to-moderate iodine deficiency is associated with lower birthweight and increased risk of preterm delivery in a large Norwegian pregnancy cohort
}

\author{
Marianne H. Abel $^{1,2}$, Ida H. Caspersen ${ }^{1}$, Verena Sengpiel ${ }^{3,4}$, Bo Jacobsson ${ }^{3,4}$, Per M. Magnus ${ }^{1}$, \\ Jan Alexander ${ }^{1}$, Helle Margrete Meltzer ${ }^{1}$ and Anne Lise Brantsaeter ${ }^{1}$ \\ ${ }^{1}$ Norwegian Institute of Public Health, Oslo, Norway, \\ ${ }^{2}$ TINE SA, Oslo, Norway, \\ ${ }^{3}$ Sahlgrenska University Hospital, Gothenburg, Sweden and \\ ${ }^{4}$ University of Gothenburg, Gothenburg, Sweden
}

\section{Abstract}

Iodine is essential in foetal development through being an integral part of the thyroid hormones. Severe iodine deficiency is associated with foetal growth restriction and preterm delivery. Less is known about the potential impact of mild-to-moderate iodine deficiency on these outcomes.

The aim of this study was to investigate whether maternal iodine intake in pregnancy was associated with birth weight (BW) z-score (i.e. BW adjusted for gestational length and sex) and preterm delivery (before week 37).

The study population included 77,995 singleton pregnancies from The Norwegian Mother and Child Cohort Study recruited in gestational week 15 in the period 2002-2008. Habitual iodine intake was calculated from a validated food frequency questionnaire covering the first half of pregnancy. Use of supplements was reported in questionnaires. Urinary iodine concentration (UIC) was measured in gestational week 18 in a subsample of $\mathrm{n}=2795$ women. Median iodine intake from food was $121 \mu \mathrm{g} / \mathrm{day}$ and median UIC was $69 \mu \mathrm{g} / \mathrm{L}$. Median UIC $<150 \mu \mathrm{g} / \mathrm{L}$ is considered insufficient in pregnant women. Median birthweight was $3610 \mathrm{~g}$ and $5.0 \%$ were born before gestational week 37 . Associations were modelled flexibly by use of restricted cubic splines, and adjusted for age, parity, prepregnancy BMI, education, smoking in pregnancy, energy intake, and fibre intake.

In non-users of iodine-containing supplements $(\mathrm{n}=48,958)$, a low habitual iodine intake from food (lower than about $150 \mu \mathrm{g} / \mathrm{day})$ was associated with a lower mean BW z-score $(\mathrm{p}<0.001)$. Compared to an intake of $150 \mu \mathrm{g} /$ day (reference), mean z-score was 0.04 SD lower at $100 \mu \mathrm{g} /$ day and $0.12 \mathrm{SD}$ lower at $75 \mu \mathrm{g}$ /day. Results were similar when using UIC as the exposure $(\mathrm{n}=2795, p=0.017)$. Any use of iodine containing supplements in pregnancy was associated with 0.03 (95\% CI: $0.01,0.04) \mathrm{SD}$ increase in BW z-score compared to no use $(\mathrm{n}=77,949, \mathrm{p}<0.001)$.

A low habitual iodine intake from food (lower than about $100 \mu \mathrm{g} / \mathrm{day}$ ) was associated with increased risk of preterm delivery $(\mathrm{p}=$ 0.003). Compared to an intake of $100 \mu \mathrm{g} /$ day (reference), $75 \mu \mathrm{g} /$ day was associated with $10 \%$ increased risk, and $50 \mu \mathrm{g} / \mathrm{day}$ with $28 \%$ increased risk. Use of an iodine-containing supplement was not associated with the risk of preterm delivery (OR: 0.97 (95\%CI: 0.91 , $1.04, p=0.42)$ ).

Inadequate iodine intake is prevalent in women of childbearing age in otherwise well-nourished populations. Our results indicate that mild-to-moderate iodine deficiency in pregnancy is associated with restricted foetal growth and increased risk of preterm delivery.

\section{Conflict of Interest}

Marianne H. Abel is employed by a Norwegian dairy company (TINE SA), and she participates in this project as an industrial Ph. D.-student financed partly by the dairy company and partly by The Research Council of Norway. This project is designed, owned and administered by The Norwegian Institute of Public Health and analysis of the data follow from protocol. All results of analysis in the project are to be published regardless of the results. The other authors have no conflicts of interest. 